D.T. Wickramasinghe, L. Ferrario, and G.V. Bicknell, eds.

\title{
Time-Resolved Optical Spectroscopy of V795 Herculis
}

\author{
Rachel J. Dickinson, Raman K. Prinja \\ Dept. of Physics \& Astronomy, University College London, Gower St, \\ London WC1E 6BT, UK
}

Simon R. Rosen, Andrew R. King

Dept. of Physics, University of Leicester, Leicester LE1 7RH, UK

Coel Hellier

Dept. of Physics, Keele University, Keele, Staffs, ST5 5BG, UK

Keith Horne

Dept. of Physics \& Astronomy, University of St Andrews, St Andrews KY16 9SS, UK

\begin{abstract}
High resolution optical spectroscopy of V795 Herculis shows complex time- and phase-dependent behaviour of the disk emission. Separate low- and high-velocity fluctuations phased on the $2.6 \mathrm{hr}$ orbital period are observed in the wings of the Balmer lines, prompting a gas stream overflow model.
\end{abstract}

\section{Introduction}

We highlight the main results from an optical spectroscopic study of the CV V795 Her, based on 6 nights of observations taken in 1994 June on the Isaac Newton Telescope $(2.5 \mathrm{~m}, \sim 1 \AA$ resolution). High signal-to-noise, phase-binned spectra, based on 241 individual exposures, were analysed. We discuss these results in terms of magnetic white dwarf and disk-overflow gas stream scenarios.

\section{Results}

The observed Balmer and He emission lines, and the He I $\lambda 4471$ absorption modulate on the $2.6 \mathrm{hr}$ orbital period. The spectra were folded into phase bins by adopting the orbital ephemeris of Shafter et al. (1990), revealing extensive differences in the morphology of the line profiles during the orbital cycle. The Balmer profiles in particular may be "decomposed" into three variable constituents: a stationary double-peaked emission core, shallow extended emission wings, and variable absorption which appears to be strongest in the first half of the cycle. The outer wing modulation in the Balmer lines is characterised by the presence of a double wave pattern with synchronised net blue-shifted and 
red-shifted humps. We argue that this variability is not due to the presence of separate waves on each wing, but arises instead from a single broad emission feature plus a superimposed (narrower) absorption component. The double-peaked inner cores have relatively fixed central velocities and do not reveal an obvious 'S'-wave variation. Doppler imaging (Marsh \& Horne 1988) suggests that the inner core Balmer emission arises from a ring of planar material located in the outer disk.

\section{Discussion}

The recent study of Casares et al. (1996) proposes a model in which the WD and companion star are phase locked, but where a partial accretion disk persists. The outer ' $S$ '-waves then arise in the magnetically entrained plasma that falls above and below the binary plane, but along the same field lines, onto the WD, with the opposed mean motions of the flows producing the net red- and blueshifted behaviour. We apply theoretical constraints to this model: to maintain synchronicity, the locking torque (which arises from the interaction of the WD's field with either an intrinsic or induced field on the companion star) must balance the accretion torque (caused by the presence of a disk). By evaluating the locking torque due to a dipole-dipole interaction and taking a reasonable range of values for the system's parameters we argue that it is essentially impossible for the WD's rotation in V795 Her to be locked to the binary motion. This argument is compounded by further objections: (i) accretion onto the upper pole from one side of the disk and onto the lower pole from the opposite side would produce anti-phased behaviour, contrary to observation, (ii) the weakness of the X-ray signal in V795 Her, and (iii) the lack of a clear X-ray modulation.

Our empirical interpretation of the data is based on the study of the emission line behaviour of PX And (Hellier \& Robinson, 1994) and other SW Sex type systems. We consider a single broad emission feature following a high-velocity 'S'-wave which has a superimposed, sympathetically phased absorption component that then yields an overall double-humped profile, the motion of which would be associated with the outer wing double 'S'-waves. This relates to a qualitative physical interpretation which invokes a disk-overflowing gas stream. The accreting gas stream overflows the outer rim of the disk, producing absorption when projected against the bright accretion disk. It reimpacts the inner disk regions, resulting in a broad emission line and a high-velocity 'S'-wave. Combining stream absorption with disk emission results in the observed double-humps of the Balmer line wings. The full paper has been submitted to MNRAS.

\section{References}

Casares, J., Martinez-Pais, I.G., Marsh, T., Charles, P.A., \& Lazaro, C. 1996, MNRAS, 278, 219

Hellier, C., \& Robinson, E.L. 1994, ApJ, 431, L107

Marsh, T.R., \& Horne, K. 1988, MNRAS, 235, 269

Shafter, A.W., Robinson, E.L., Crampton, D., Warner, B., \& Prestage, R.M. $1990, \mathrm{ApJ}, 354,708$ 